\title{
References
}

Grundy DJ, Fellows GJ, Gillett AP, et al. 1983 A comparison of fine-bore suprapubic and an intermittent urethral catheterisation regime after spinal cord injury. Paraplegia 21:227-232.

LLOYD LK, KUHLEMEIERE KU, FINE PR, et al. 1986 Initial bladder management in spinal cord injury-does it make a difference? Fournal of Urology 135:423-527.

NAMiki T, Ito H, YASUDA K 1978 Management of the urinary tract by suprapubic cystostomy kept under a closed and Aseptic state in the acute stage of the patients with a spinal cord lesion. Fournal of Urology 119:359-362.

PeAtField RC, BuRT AA, SMITH PH 1983 Suprapubic catheterisation after spinal cord injury: a follow-up report. Paraplegia 21:220-226.

Wyndaele JJ, De Sy WA, Claessens H 1982 Early urological complications in spinal cord injury patients treated with a Foley catheter. Acta Urologica Belgica 50:335-342.

Wyndaele JJ, De Sy WA, Claessens H 1985 Evaluation of different methods of bladder drainage used in the early care of spinal cord injury patients. Paraplegia 23:18-26.

\section{Reply from Dr Friedhelm Noll}

The majority of spinal cord injured patients need initial treatment in an intensive care unit, requiring continuous fluid balancing, frequently done with indwelling Foley catheters. Here the SPC is of definite advantage, combining continuous drainage, simplicity of care and low infection rate with the absence of all disadvantages of Foley drainage.

Our study showed that the probability of entering the next ISC or CIC phase with sterile urine is higher with SPC than with intermittent catheterisation. First infection is usually dependent on sterility performed during insertion of SPC, which is done, in our setting, by the physicians of the SCI unit, giving us control over initial sterility.

Early clean or sterile intermittent catheterisation is more favorable than any kind of continuous drainage. SPC should be left in place only during the acute phase of spinal cord injury. Then patients should be changed progressively to CIC or ISC. In this regard, our study does not intend to replace intermittent catheterisation, but introduces another safe method for early bladder management.

Certainly the work of Guttmann and Frankel demonstrated, that intermittent catheterisation is a reliable tool, to decrease urological problems during the first stage of medical rehabilitation after SCI. Indeed, we believe, that intermittent catheterisation is one of the best bladder managements, when it is combined with low pressure bladder function.

The technique of bladder drainage alone does not change bacteriological resistances in a ward; this is dependent on multiple factors, one of those is certainly nursing care. SPC drainage in the acute phase avoids problems secondary to inappropriate intermittent catheterisation or Foley catheter drainage, during a phase, when patients are particularly vulnerable.

If infection occurred, it could be cured without major difficulty, even with SPC in place, assuming, that SPC drainage is done less than 2 weeks and first infection is meant. In prolonged SPC drainage or multiple reinfections, antibiotic treatment faces the same problems as known from indwelling catheters, still avoiding urethral problems.

In short: SPC is a safe management during the initial phase of SCI. It is superior to Foley drainage, considering side effects and long term results, and is easy to use. The probability of infection is reduced and almost independent of 
care during the first phase, when patients have to be kept in the intensive care unit. When clinically stable patients should be changed to ISC or CIC. Focussing bladder management on pressure results in a more favourable urological outcome. To achieve and maintain low pressures, in the majority of reflex bladders, medication is necessary. Oxybutynin and Imipramine have proved to be reliable anticholinergic agents with good suppressive effects on bladder activity. SPC as an initial management does not delay recovery of bladder function, nor does it interfere with the described bladder management. 